\title{
Design, Identification and Control of a Fast Nanopositioning Device
}

\author{
S. Salapaka ${ }^{1 \dagger}$, A. Sebastian ${ }^{2 \ddagger}$, J.P. Cleveland ${ }^{3 \S}$ and M.V. Salapaka ${ }^{4 \ddagger}$ \\ ${ }^{1}$ salpax@engineering.ucsb.edu, ${ }^{2}$ abuseb@iastate.edu, ${ }^{3}$ jason@asylumresearch.com and ${ }^{4}$ murti@iastate.edu \\ †Department of Mechanical and Environmental Engineering, UCSB, Santa Barbara, CA 93106 \\ ${ }^{\ddagger}$ Department of Electrical and Computer Engineering, Iowa State University, Ames, IA 50014 \\ ${ }^{\S}$ Asylum Research, 601-C Pine Avenue, Goleta, CA 93117
}

\begin{abstract}
This paper presents the design, identification and control of a nanopositioning device. The device is actuated by a piezoelectric stack and its motion is sensed by a Linear Variable Differential Transformer (LVDT). A fourth order single input single output model has been identified to describe its dynamics. It is demonstrated that PI control law does not meet the bandwidth requirements for positioning. This motivated the design and implementation of an $\mathcal{H}_{\infty}$ controller which demonstrates substantial improvements in the positioning speed and precision besides eliminating the undesirable nonlinear effects of the actuator. The characterization of the device in terms of bandwidth, resolution, and repeatability is also shown.
\end{abstract}

\section{Introduction}

The advent of new techniques to explore properties of near atomic-scale structures has led to the development of the new field of nanotechnology. In the past decade, it has become evident that nanotechnology will make fundamental contributions to science and technology. Inevitably, most schemes of nanotechnology impose severe specifications on positioning. This demand of ultra-high positioning precision forms a pivotal requirement in many applications of nanotechnology. For example micro/nanopositioning systems are essential in auto focus systems [1] in optics; disk spin stands and vibration cancellation in disk drives [2]; wafer and mask positioning in microelectronics [3]; piezo hammers [4] in precision mechanics; and cell penetration and micro dispensing devices [5] in medicine and biology. Besides this requirement of high precision, there is also an increasing need for high bandwidth positioning systems. For example in the field of cell biology, there are attractive proposals to employ nano-probes to track events in the cell. These events often have time scale in microsecond or nanosecond regimes. This necessitates high bandwidth positioning systems.

To meet the dual goal of high precision positioning at high bandwidth, novel sensors and actuators have been studied and developed. Most of the current high precision positioning devices utilize piezoelectric materials for actuation. The crystal lattices of these materials deform on the application of an electric field and these deformations are used for positioning with high accuracy. However, the precision in positioning is significantly reduced due to nonlinear hysteresis effects especially when the piezoactuators are used in rela- tively long range positioning applications. For instance, the maximum error due to hysteresis can be as much as $10-15 \%$ of the path covered. Another cause for the loss in precision is the drift due to creep effects. These effects become noticeable when piezoactuation is required over extended periods of time, i.e. during slow operation modes of the positioning device. Most of the commercially available devices circumvent these nonlinear effects at the cost of their performance by restricting the devices to low drive applications, where the behaviour is approximately linear and/or limiting their motions to some specific trajectories, for which nonlinear effects have been accounted for and appropriately compensated.

In the recent past, some efforts have been made to address these nonlinear effects. In [6], charge control (in contrast to voltage control) has been proposed to diminish the hysteresis effects. However these techniques lead to increased drift and saturation problems and lead to further reduction of the travel range and the positioning bandwidth of the piezoactuator. In [7], post-correction techniques for removing creep and hysteresis effects from SPM-images have been presented. However, these post-processing methods cease to be useful for applications (such as cell biological studies) in which real time compensation is needed. In [8], the design of a feedback controller using an optical sensor attachment to a commercial AFM to enhance its performance has been described. The feedback laws have been demonstrated to eliminate these nonlinear effects and considerably increase the positioning bandwidths. In [9], the problem of nonlinear effects has been addressed by a careful identification/modeling of these nonlinearities and then using a model based inversion approach to compensate for their adverse effects. However, the efficacy of this approach depends on the extent of accuracy of modeling of the nonlinearities. But, this design methodology can be used in conjunction with the feedback design to achieve better performance and also robustly account for modeling uncertainties.

In this paper, we present a nanopositioning device with a piezoelectric actuator and a Linear Variable Differential Transformer (LVDT) sensor. The device described here is an independent unit, and is not a modification or an enhancement of an already existing device. The piezoactuator used here is a stack-piezo, in contrast to piezoelectric tube in [8] and [9], which has better hysteresis and creep properties but is much more expensive. We also show that the traditional and commonly used Proportional-Integral $(P I)$ control laws in the scanning probe community have a highly unsatisfactory performance. This motivated us to 
carry out an $\mathcal{H}_{\infty}$ control design, which gave significantly better results. For instance, we were able to obtain bandwidths as high as $130 \mathrm{~Hz}$ by this design in contrast to less than $3 \mathrm{~Hz}$ with the $P I$ control law.

\section{Device Description}

A schematic of the device is shown in Figure 1. It consists of a flexure stage with a sample holder, an evaluation stage, an actuation system, a detection system and a control system.

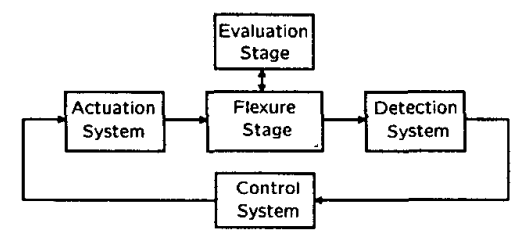

Figure 1: A schematic block diagram of the device.



(a)

(b)

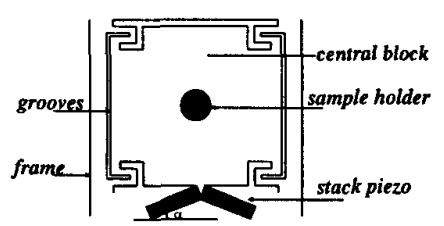

(c)

Figure 2: (a) The base plate of the flexure stage. (b) The exploded view of the flexure and evaluation stages.

The flexure stage consists of two components: the base plate and the top plate. The base plate (see Figure 2(a)) is $20 \mathrm{~cm} \times 20 \mathrm{~cm} \times 5 \mathrm{~cm}$ and is made of steel. From its centre protrudes a cylindrical block (the sample holder) which has a provision for the sample to be placed on it. This part of the base plate that seats the sample holder executes motion relative to its periphery. This motion is obtained by the serpentine spring design (see Figure 2(c)) where design grooves (of about $150 \mu \mathrm{m}$ wide) are cut in the base plate that make it possible for the central block to move relative to the frame. The top plate sits on the base plate and is of similar dimensions as the base plate. It provides the sample an access to the evaluation stage (see Figure 2(b)). The evaluation stage consists of a modified Atomic Force Microscope head where the features on the sample cause the cantilever to deflect in vertically as the sample moves under its tip. The resulting deflection signal is processed to infer the topography of the surface.

The actuation system consists of a voltage amplifier and a piezo-stack arrangement. This arrangement sits in the slot in the flexure stage (see Figure 2(a)). The piezo deformation imparts the motion to the central block of the flexure stage. The input to the amplifier (which has gain of -15 ) is restricted to be negative and to be less than $10 \mathrm{~V}$ in magnitude since the piezo-stack saturates beyond this limit. The piezo stacks are at an angle $\alpha \approx 7.5$ degrees (see Figure 2 ) because this arrangement, besides providing the sufficient force to the central block, also achieves a mechanical gain of $1 / \sin (\alpha)$.

The detection system consists of a LVDT and associated demodulation circuit. It has a resolution in the order of a few angstroms. The LVDT has been adjusted so that it outputs $0 V$ when an input of $-5 V$ is given to the actuation system. The corresponding position of the central block is called the null position. The LVDT output signal is proportional to the motion of the central block.

The design of the of the control laws have been presented in Section 3. These laws were implemented on a Texas Instruments $\mathrm{C} 44$ digital signal processor.

\section{Identification of the system}

The modeling of the device was done using the black-box identification technique where we chose a specific point in the operating range of the device (where its behaviour is approximately linear) and obtain a model of the device at this point by studying its frequency response over a prespecified bandwidth. For this purpose, we used a HP signal analyzer, which gave a series of sinusoidal inputs, $u=-5+A \sin (\omega t)(V)$, with frequencies spanning over a bandwidth of $2 \mathrm{kHz}$. The amplitude of the signals, $A$ were chosen small enough $(\leq 50 \mathrm{mV})$ to be in the linear regime of the device. The frequency response of the plant $G$ at the null point was then obtained by recording steady state values of the output $y$ for each $u$ (see Figure 3). Accordingly, a fourth order non minimum phase transfer function:

$$
G(s)=\frac{9.7 \times 10^{4}\left(s-(7.2 \pm 7.4 i) \times 10^{3}\right)}{\left(s+(1.9 \pm 4.5 i) \times 10^{3}\right)\left(s+(0.12 \pm 15.2 i) \times 10^{2}\right)}
$$

was fit to this data. Figure 3 shows that there is a good match between this frequency response data and the one simulated from the model, $G(s)$.

\section{Control Design}

The model inferred for the device at the null position was employed to design the feedback laws. The schematic of the closed loop system is shown in Figure 4. Here $e=S r-T n$ is the error signal where $S=(1+G K)^{-1}$ is the sensitivity function and $T=(1+G K)^{-1} G K$ is the complementary sensitivity function. The primary objective of the control design is to achieve good tracking with high bandwidths. Also, the feedback laws were constrained to provide control signals that were negative and within actuator saturation limits $(-10 \mathrm{~V}$ to $0 \mathrm{~V})$. Besides these implementation constraints, the presence of RHP zeros imposes fundamental 


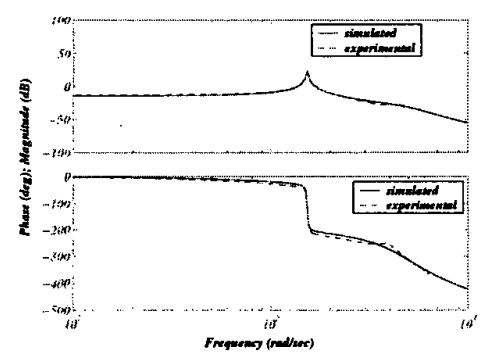

Figure 3: A comparison of experimentally obtained and simulated frequency responses of the plant.

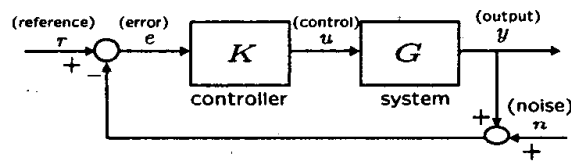

Figure 4: A schematic block diagram of the closed loop system.

constraints. For example, the classical root locus analysis predicts high gain instability of the system. This rules out high gain feedback laws. They also impose a fundamental limit on the achievable bandwidth of the closed loop system. It has been shown in $[10,11]$, that a complex pair of RHP zeroes, $z_{1,2}=x \pm i y$ (as in this case $z_{1,2}=(1.72 \pm 7.36 i) \times 10^{3}$ ), the "ideal" controller leads to the following sensitivity function,

$$
S=\frac{4 x s}{(s+x+j y)(s+x-j y)} .
$$

By the above criteria the achievable bandwidth (the frequency at which $|S(j \omega)|$ crosses $-3 d B$ from below) is approximately $415 \mathrm{~Hz}$ for the system. This controller is "ideal" in the sense that it may not be realizable and which, for a unit step reference $r(t)$, generates an input $u(t)$ which minimizes the integral square tracking error:

$$
\int_{0}^{\infty}|y(t)-r(t)|^{2} d t
$$

In industry, it is a common practice to design proportional $(P)$ or proportional-integral $(P I)$ controllers. In the next section, we show that these controllers do not perform well and it becomes. inevitable to look for more sophisticated designs. In particular, we present $\mathcal{H}_{\infty}$ control design which shows a great improvement over $P$ and $P I$ controllers.

$P$ and $P I$ controller designs: From the analysis of the root-locus plot of the open loop system, it is seen that the closed loop system is unstable for feedback gains greater than 0.1674 which rules out the use of proportional control.

In the design of $P I$ control law $\left(k_{p}+k_{i} / s\right)$, since we know the structure of the controller, we can determine the regions in $k_{p}-k_{i}$ plane which guarantee closed loop stability. Figure 5 (a) shows this plot. It should be noted that the region (in the $k_{p}-k_{i}$ ) that gives high bandwidth (see plot (d)) is the region with low gain margin (see plot (c)), i.e. there is a trade-off between robustness and performance. So we chose $k_{p}=0.01$ and $k_{i}=75$ which guarantees a gain margin of 1.57 and a phase margin of $90^{\circ}$ and the corresponding bandwidth of the closed loop transfer function is $2.12 \mathrm{~Hz}$. It
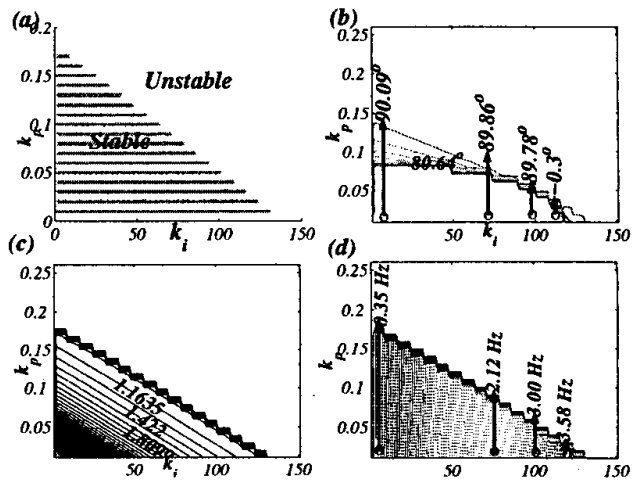

Figure 5: (a) The region in $k_{i}-k_{p}$ plane that guarantees closed loop stability. The contour plots showing (b)the phase margins (c) the gain margins (d) the bandwidths of the closed loop systems corresponding to different points in the $k_{i}-k_{p}$ plane.

should be noted that the bandwidth $(<3 \mathrm{~Hz})$ attained here is much less than the achievable bandwidth $(\approx 415 \mathrm{~Hz})$ as described in the previous section.

$\mathcal{H}_{\infty}$ controller design: The main advantage of using this design is that it includes the performance objectives in the problem formulation itself. In this setting, the $\mathcal{H}_{\infty}$ optimal control problem amounts to finding the control feedback law, $K$, such that $\left\|P_{z w}\right\|_{\infty}$ is infimized, where $P_{z w}$ is the transfer function from $w$ to $z$. The first step towards

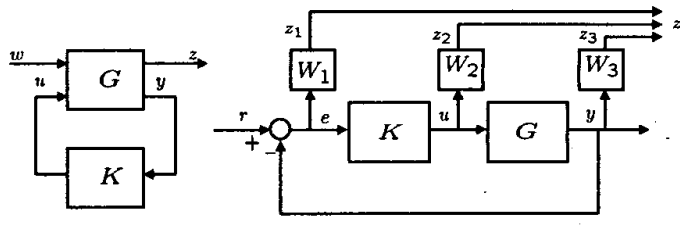

(a)

(b)

Figure 6: (a) The generalized plant framework. (b) The closed loop system with regulated outputs.

$\mathcal{H}_{\infty}$ control design is to form the generalized plant, $P$. In the system (see Figure 6(b)), the exogenous input $w$ is the reference signal $r$, the control input is $u$ and the measured output $v$ is the error signal $e$. In order to reflect the performance objectives and physical constraints, the regulated outputs were chosen to be the weighted transfer function, $z_{1}=W_{1} e$, the weighted system output, $z_{2}=W_{2} y$ and the weighted control input, $z_{3}=W_{3} u$. This choice of out-

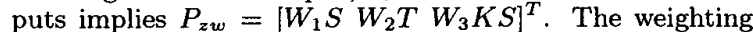
functions $W_{i}, i=1,2$ and 3 are used to scale these closed loop transfer functions to specify the frequency information of the performance objectives and system limitations. The 
transfer function, $W_{1}$, is chosen such that its inverse (an approximate upper bound on sensitivity function) has a gain of $0.1 \%$ at low frequencies $(<1 \mathrm{~Hz})$ and a gain of $\approx 5 \%$ around $200 \mathrm{~Hz}$. It was chosen to be a first order transfer function, given by

$$
W_{1}(s)=\frac{0.1667 s+2827}{s+2.827} .
$$

This weighting function puts a lower bound on the bandwidth of the closed loop system but does not specify the roll of the open loop system to prevent high frequency noise amplification and limiting the bandwidth to be below Nyquist frequency. To do this, we scale the complementary sensitivity function, $T$, by

$$
W_{2}=\frac{s+235.6}{0.01 s+1414}
$$

which has low gains at high frequencies and vice versa. The transfer function, $K S$ was scaled by a constant weighting $W_{3}=0.1$ to restrict the magnitude of the input signals such that they are within the saturation limits. This weighting constant gives control signals that are at most six times the reference signals.

In practice it is not usually necessary to obtain an optimal controller and often it is computationally simpler to design a suboptimal one (i.e. one close to optimal one in the sense of $\mathcal{H}_{\infty}$ norm). In particular, for any $\gamma>\gamma_{\text {opt }}>0$ we can find a controller transfer function $K$ such that $\left\|P_{z w}\right\|_{\infty} \|<\gamma$ where $\gamma_{\text {opt }}$ is the optimal value. The controller is designed (using the function, hinfsyn in MATLAB) for $\gamma=2.415$ and the weighting functions described above. The following sixth order controller transfer function, $K(s)$ was obtained with a DC gain of $2.2599 \times 10^{3}$, its poles at $-1.14959 \times$ $10^{7},-1.4137 \times 10^{5},-5.6432 \times 10^{3},-2.8274$ and $(-1.5676 \pm$ $5.8438 i) \times 10^{3}$, and its zeroes at $-1.4137 \times 10^{5},(-1.8647 \pm$ $4.4958 i) \times 10^{3}$ and $-1.1713 \times 10^{1} \pm 1.5205 i \times 10^{3}$.

The corresponding controller and the sensitivity transfer functions are shown in Figure 7. The bandwidth of the sensitivity transfer function is found to be $138 \mathrm{~Hz}$. It should be noted that this is an enormous improvement over the PI controller. Also, this controller provides a gain margin of 2.57 and a phase margin of $62.3^{\circ}$ as opposed to the values of 1.57 and $\approx 89^{\circ}$ in the PI controller. In this figure, we have compared this sensitivity function with the "ideal" one introduced earlier in the section.

Figure 8 shows the performance of this controller for a 25,50 and $100 \mathrm{~Hz}$ triangular and $100 \mathrm{~Hz}$ reference signals. It can be seen that the closed loop system tracks well the $25 \mathrm{~Hz}$ and $50 \mathrm{~Hz}$ signals (see plots (a) and (b)). The mismatch in the case of the $100 \mathrm{~Hz}$ (in (c)) signal is due to the accentuation of higher modes of the triangular wave. In contrast, the $100 \mathrm{~Hz}$ sinusoidal signal shown in (d) does not have higher harmonics and the closed loop system shows much improvement in its tracking.

\section{Characterization of the device}

In this section, we characterize the closed loop and the open loop device in terms of its resolution, bandwidth, and the range. First, the device was calibrated using a calibration sample which had $180 \mathrm{~nm}$ high grooves every $5 \mu \mathrm{m}$. This grid was placed on the sample holder and probed by the evaluation stage. A triangular input of amplitude $2 \mathrm{~V}$ was
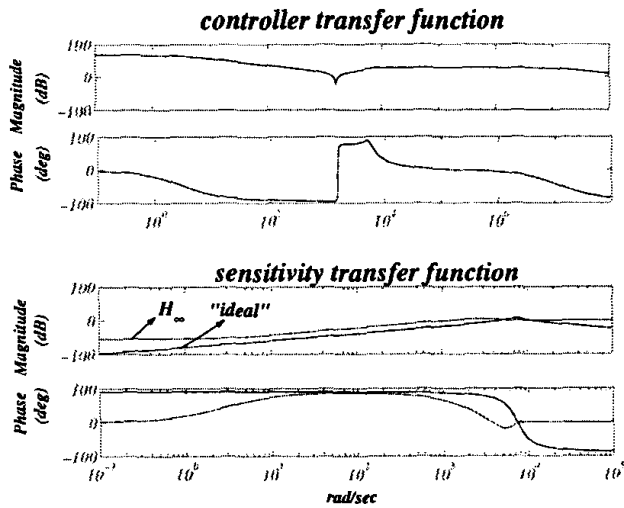

Figure 7: The controller and the sensitivity transfer functions

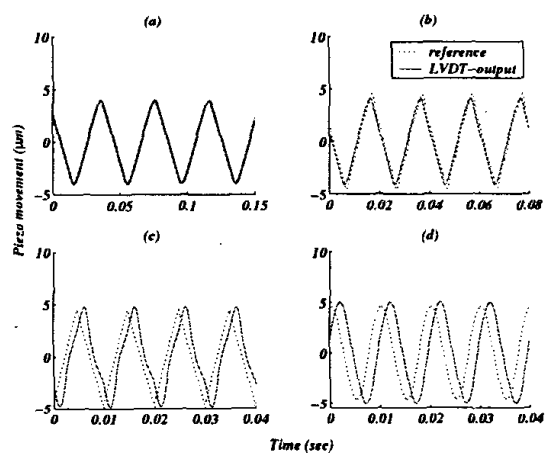

Figure 8: Tracking of (a) $25 \mathrm{~Hz}$ (b) $50 \mathrm{~Hz}$ (c) $100 \mathrm{~Hz}$ triangular waves and (d) $100 \mathrm{~Hz}$ sinusoidal wave using the $\mathcal{H}_{\infty}$ controller.

given and the resulting LVDT output showed the presence of 7.26 grooves. This implies the device has a static sensitivity of $18.15 \mu \mathrm{m} / \mathrm{V}$.

As already mentioned, piezoactuators do not have any backlash or friction and therefore have very fine resolutions. The resolution of the device, therefore, depends on the experimental environment and it is limited by thermal and electronic noise. Since the effect of the noise increases with the bandwidth, finer resolutions are expected at lower bandwidths. To determine the resolution at different bandwidths, $\omega_{b}$, we gave a constant input of $-5 V$ to the actuation system and recorded the resulting noise signal from the LVDT (the detection system). The resolution was defined as three times the standard deviation $\sigma$ of this LVDToutput signal. The standard deviations at different bandwidths were obtained by calculating the area under the Power Spectral Density $(S(\omega))$ curve up to the corresponding bandwidths, therefore, resolution $=3\left(\int_{0}^{\omega_{6}} S(\omega) d \omega\right)^{\frac{1}{2}}$.

The experiments to determine resolution were done both in open and closed loop configurations. The advantages of closing the loop is that, in this case the creep and drift effects are compensated and thus better resolutions are 
obtained (see Figure 9). As seen in the previous section (see Figure 7), the $\mathcal{H}_{\infty}$ controller achieved an approximate bandwidth of $138 \mathrm{~Hz}$, however, for slower (low bandwidth) application, one can decrease the bandwidth appropriately and achieve high resolutions. It was seen that the input voltage of approximately $4 \mathrm{~V}$ can be given without reaching the limits of the actuator. This guarantees a travel range of $70 \mu \mathrm{m}$

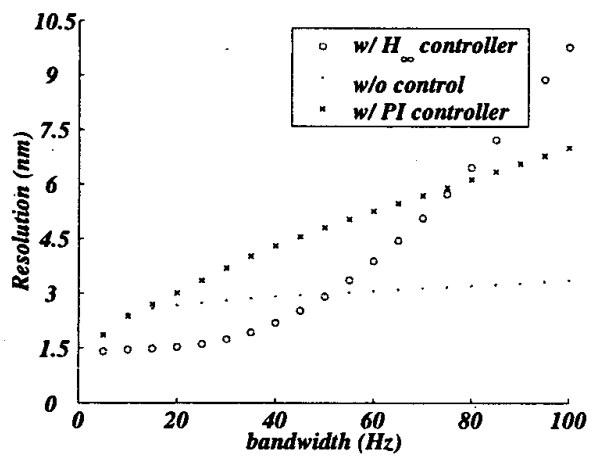

Figure 9: The comparison of resolution vs bandwidth plots between open loop and closed loop with PI and $\mathcal{H}_{\infty}$ controllers.

Elimination of nonlinear effects

The positioning precision of the piezoactuators is significantly reduced due to nonlinear effects such as hysteresis, drift and creep. Hysteresis effects are significant when the sensors are used for relatively long ranges. Therefore piezoactuators are typically operated in linear ranges to avoid positioning effects. However, with the feedback control laws, these nonlinear effects are compensated and thus the closed loop device does not show any hysteresis. To study this, we first plotted hysteresis curves by operating the device in open loop and then compared them with the curves obtained by repeating the experiment in the closed loop. Input signals (less than $1 \mathrm{~Hz}$ triangular pulses) of increasing amplitudes ( $1 \mathrm{~V}$ to $4 \mathrm{~V}$ ) were given in open loop and the corresponding output signals recorded (see Figure 10). It is observed that the hysteresis effects are dominant in higher amplitudes (longer travels) and become smaller as the travel lengths are reduced. The hysteresis is quantified numerically in terms of maximum input (or output) hysteresis usually given as a percentage of the full scale. Figure 10 shows that the feedback control laws virtually eliminate all hysteretic effects, and the output and the reference signals match well.

Creep is another undesirable nonlinear effect common with piezoelectric actuators. It is related to the effect of the applied voltage on the remnant polarization of the piezo ceramics. If the operating voltage of a piezoactuator (open loop) is increased (decreased), the remnant polarization (piezo gain) continues to increase (decrease), manifesting itself in a slow creep (positive or negative) after the voltage change is complete. This effect is approximately described by the equation,

$$
y(t) \approx y_{0}\left(1+\gamma \log \left(t / t_{0}\right)\right),
$$

where $t_{0}$ is the time at which the creep effect is discernible, $y_{0}$ is the value of the signal at $t_{0}$ and $\gamma$ is a constant, called

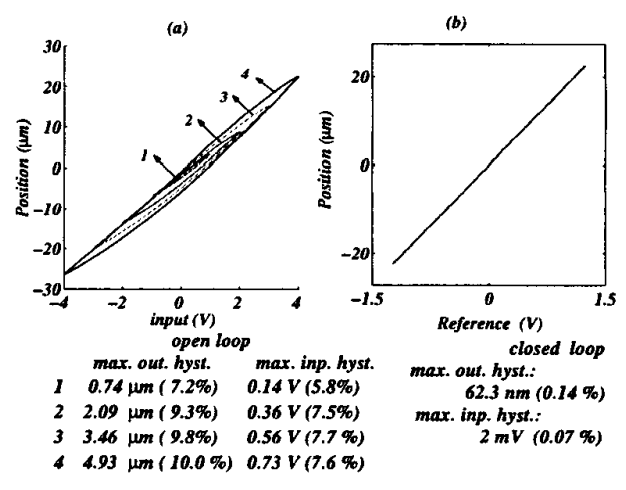

Figure 10: Hysteresis in (a) the open loop configuration, (b) its elimination in the closed loop configuration.

the creep factor, that characterizes this nonlinear effect. To measure this effect, we studied a step response of the device in open and closed loop configurations. We see that the output in the open loop case responds to the reference signal but instead of reaching a steady state value it continues to decrease at a very slow rate. The response $y(t)$ was found to approximately satisfy the creep law with a creep factor of 0.55 The same experiment conducted with the closed loop shows that the feedback laws virtually eliminate this effect and the system tracks the reference signal exactly.

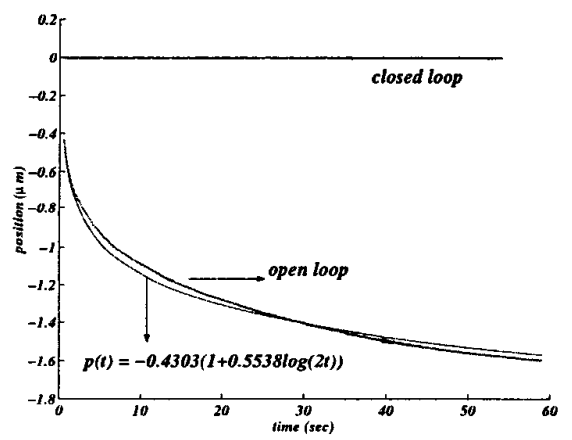

Figure 11: (a) responses to the step input of open loop and closed loop systems (b) The elimination of creep in the closed loop system and its approximation by a creep law.

A significant adverse effect of the nonlinearities in the open loop is that of non repeatability. This was seen clearly in the calibration experiment described in the previous section. In the open loop case, the grooves that were observed when traveling in one direction were not concomitant with those in the other direction. This has been shown in Figure 12(a). The corresponding hysteresis curve is shown in (c). These effects were removed with feedback control and in (b), we see that the grooves in the forward and reverse direction fall exactly on top of each other. The corresponding hysteresis plot is shown in (d). The mismatch in the open loop is more clearly seen in Figure 13(b) where the image obtained in one direction is kept behind the one got in the other direction 

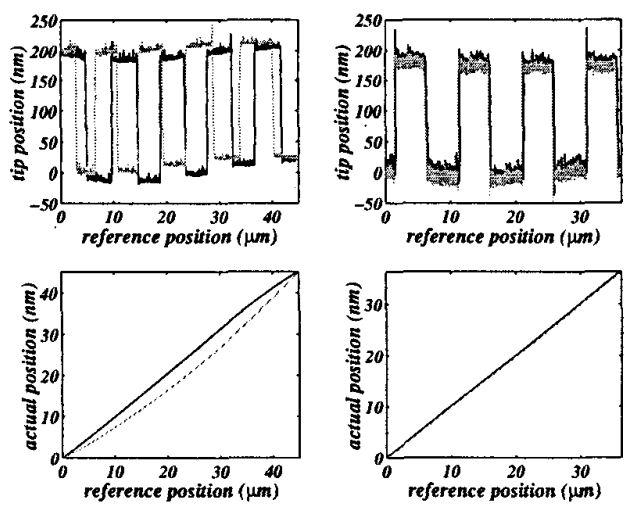

Figure 12: Evaluation stage results: (a),(c) mismatch in the position of grooves between the forward and the backward traverses in the open loop and the corresponding hysteresis cycle. (b), (d) a good match in the closed loop configuration and the corresponding hysteresis cycle.

for the sake of comparison. In (c), the near perfect match with the closed loop system is demonstrated.

\section{Conclusions}

This paper presents the design, modeling and characterization of a fast high-precision nanopositioning device. It was demonstrated that $P I$ control laws were not satisfactory for fast $(>3 \mathrm{~Hz})$ tracking which necessitated an $\mathcal{H}_{\infty}$ design. These laws were implemented and the experimental results demonstrate substantial improvements in the positioning precision of and the operating speeds $(\approx 130 \mathrm{~Hz})$. Also, since these undesirable nonlinear effects are generic to most piezoelectric actuated devices, this control methodology can be adapted to them to obtain higher precision and larger bandwidths. Our future goal is to modify this device to have capability to position in all the three directions. This can be done by modifying the existing serpentine-spring design and having an identical actuator-flexure stage-sensor arrangement at right angles to the existing one (in $x$-direction) to provide for motion in the other lateral direction (along $y$ axis); and by replacing the cylindrical block in the flexure stage by a piezoelectric material to allow for moving the sample in the vertical $(z)$ direction. Once these modifications are done, this device can be used as a microscope to image surfaces of various samples and study their properties.

\section{References}

[1] Z. Rihong, Daocai X., Y. Zhixing, and C. Jinbang. Research on systems for measurements of CCD parameters. In Proceedings of the SPIE- the International Society for Optical Engineering, volume 3553, pages 297-301, September 1998 .

[2] M. Jianxu and M.H. Ang Jr. High-bandwidth macro/microactuation for hard disk drive. In Proceedings of the SPIE- the International Society for Optical Engineering, Boston, volume 4194, pages 94-102, November 2000.

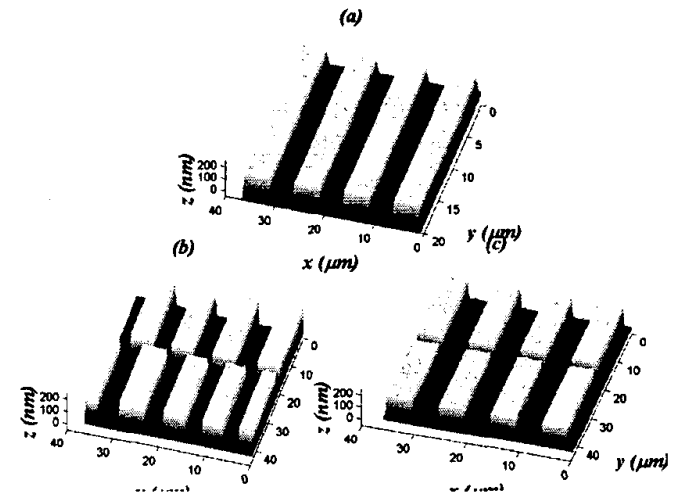

Figure 13: (a) The reference (calibration sample) geometry. (b) The mismatch in the position of grooves between the forward and the backward traverses in the open loop. (c) a good match in the closed loop configuration.

[3] D.L. White and O.R. Wood. Novel alignment system for imprint lithography. In Jurnal of Vacuum Science $\mathcal{B}$ Technology B (microelectronics and Nanometer structures), Rancho Mirage, volume 18(6), pages 3552-3556, May-June 2000.

[4] C.K. Lee, C.T. Lin, C.C. Hsia, and W.C. Liaw. New tools for structural testing: piezoelectric impact hammers and acceleration rate sensors. Journal of Guidance, Control and Dynamics, 21(5):692-697, September-October 1998.

[5] D.R. Meldrum, W.H. Pence, S.E. Moody, D.L. Cunningham, M. Holl, P.J. Wiktor, M. Saini, M.P. Moore, L-S Jang, M. Kidd, C. Fisher, and A. Cookson. Automated, integrated modules for fluid handling, thermal cycling and purification of dna samples for high throughput sequencing and analysis. In Proceedings of 2001 IEEE/ASME International conference on Advanced Intelligent Mekatronics, volume 2, pages 1211-1219, July 2001.

[6] H. Kaizuka. Application of capacitor insertion method to scanning tunneling microscopes. Rev. of Sci. Instrum., 60(10):3119-3122, 1989.

[7] R.C. Barrett and C.F. Quate. Optical scan-correction system applied to atomic force microscopy. Rev. of Sci. Instrum., 62:1393-1399, 1991.

[8] A. Daniele, S. Salapaka, M.V. Salapaka, and M. Dahleh. Piezoelectric Scanners for Atomic Force Microscopes:Design of Lateral Sensors, Identification and Control. In Proceedings of the American Control Conference, San Diego, California, pages 253-257, June 1999.

[9] D. Croft, G. Shedd, and S. Devasia. Creep, Hysteresis and Vibration compensation for Piezoactuators: Atomic Force Microscopy Application. In Proceedings of the American Control Conference, Chicago, Illinois, pages 2123-2128, June 2000.

[10] M. Morari and E. Zafiriou. Robust Process Control. Prentice-Hall, Englewood Cliffs, 1989.

[11] S. Skogestad and I. Postlethwaite. Multivariable Feedback Control, Analysis and Design. John Wiley and Sons, 1997. 